\title{
LOS TURISMOS DE INTERIOR EN GALICIA. BALANCE Y PERSPECTIVAS
}

\author{
Xoxé M. SANTOS SOLLA \\ Departamento de Geografía. Universidad de Santiago de Compostela
}

Recibido: 08/11/2011

Devuelto: 07/06/2012

Aceptado: 13/07/2012

RESUMEN: A partir de la década de 1990, los turismos de interior en Galicia empiezan a despertar un inusitado interés. A la oferta, bastante poco organizada, de algunos puntos del litoral gallego se une un número creciente de establecimientos rurales con una importante financiación pública. Además, la puesta en valor del Camino de Santiago abre nuevas expectativas para estos territorios. Posteriormente, el termalismo, la incorporación de pequeñas ciudades o la apertura de establecimientos singulares, a veces bajo la denominación de hoteles monumento, junto con programas en destino u otros de desarrollo rural, generan esperanzas de que el turismo sea realmente una actividad que transforme las dinámicas recesivas de estos espacios.

Casi dos décadas más tarde, el balance y las perspectivas de futuro son bastante desiguales. El turismo rural pasó de ser prioritario a convertirse en una oferta secundaria con graves problemas por causa de unas tasas de ocupación extremadamente bajas. Los esfuerzos por crear producto que lo haga más atractivo no parece que estén dando los frutos esperados. El termalismo, así como otras tipologías, más que crear sinergias generan competencias. Finalmente el Camino de Santiago a pesar de su indudable éxito tampoco favoreció el desarrollo de estos espacios, sólo de forma puntual.

El futuro en principio no parece demasiado halagüeño. Creemos que se ha perdido la perspectiva del turismo como fenómeno que genera desarrollo y se ha desvinculado de forma creciente del mundo rural, que es simplemente un soporte físico para llevar adelante una actividad económica.

PALABRAS CLAVE: turismo interior, Galicia, desarrollo, rural.

\section{RURAL TOURISM IN GALICIA. ASSESSMENT AND PERSPECTIVES}

ABSTRACT: Since the 1990s inland tourism started to arouse an unusual amount of interest in Galicia. The rather disorganized offer which could be found at some coastal sites throughout Galicia was now combined with an increasing number of rural hotels and businesses receiving significant public funding. Furthermore, the considerable momentum gained by the Camino de Santiago created rising expectations for these areas. Later on thermalism, the incorporation of small towns and the setting up of singular businesses -some of them known as hotel monumento-, together with local destination and rural development plans, would raise new hopes of tourism actually becoming an activity which might transform the recessive dynamics of these territories. 
Nearly twenty years after, both the balance and prospects for the future are quite unequal. Rural tourism stopped being a priority to become a sort of secondary offer facing serious problems related to extremely low occupancy rates. Efforts to create a product which may make this kind of tourism more attractive do not seem to be producing the results expected. Rather than synergies, thermal and other types of tourism are leading to intense rivalry. And finally, despite its overwhelming success, the Camino de Santiago did not really stimulate other than a momentary development of these inland areas.

The prospects are -at least in principle- not very encouraging. We believe that the perspective of tourism as a driver of development has been lost, and that it has gradually distanced itself from the rural world, which has become no more than a simple physical support to undertake a business activity.

KEY WORDS: inland tourism, Galicia, development, rural.

\section{INTRODUCCIÓN}

Fue hacia finales de la década de 1980 y sobre todo a principios de la de 1990 cuando los turismos de interior, y fundamentalmente el denominado turismo rural, empezaron a ser objeto intenso de atención, tanto por parte de responsables públicos como de la academia (FORNEAU, 1984; BOTE, 1984, 1985, 1987 y 1988; FLORES y BOTE, 1987; BARDÓN, 1987; GALIANO, 1991; REYNA, 1992; CROSBY, 1993; CALS et AL., 1995). Esta creciente muestra de interés en España fue consecuencia de la conjunción de toda una serie de factores como: la crisis rural y su búsqueda de soluciones; la contención del productivismo incitada por la Unión Europea y la necesaria introducción de otro tipo de actividades; el supuesto final de ciclo del turismo de sol y playa que daba oportunidades a nuevos espacios que se habían mantenido al margen del mismo; o la descentralización de la política turística que abre nuevas vías a los territorios de interior, hasta entonces básicamente ajenos a este fenómeno.

A partir de ese momento todas las comunidades autónomas, unas antes y otras después, comenzaron a impulsar el turismo rural. En un principio se trató fundamentalmente de abrir establecimientos de alojamiento en los que se valoraba sobre todo la singularidad y la tipicidad de los mismos, incluso por encima de los servicios ofertados, rompiendo claramente con los sistemas tradicionales de clasificación; característica que por cierto se mantiene en la nueva Ley de Turismo de Galicia (2011). La financiación para la puesta en valor de estos espacios vino de la mano de programas europeos de desarrollo rural, así como de iniciativas propias de las Comunidades Autónomas.

Los resultados fueron bastante sorprendentes en cuanto a la creación de alojamiento con un crecimiento muy significativo en el número de establecimientos y de plazas, acompañado siempre de nuevos marcos legales para regular esta actividad. 
Cuadro 1. Número de plazas estimadas en alojamiento de turismo rural

\begin{tabular}{lrrr}
\hline & 2001 & 2010 & \% de incremento \\
\hline Andalucía & 2.074 & 10.945 & 427,7 \\
Aragón & 4.610 & 8.555 & 85,5 \\
Asturias & 2.813 & 12.406 & 341,0 \\
Baleares & 1.863 & 4.696 & 152,0 \\
Canarias & 1.636 & 4.119 & 151,7 \\
Cantabria & 3.222 & 7.646 & 137,3 \\
Castilla y León & 7.471 & 28.359 & 279,5 \\
Castilla-La Mancha & 2.498 & 9.916 & 296,9 \\
Cataluña & 5.533 & 13.223 & 138,9 \\
Comunidad Valenciana & 2.981 & 9.151 & 206,9 \\
Extremadura & 997 & 5.674 & 469,1 \\
Galicia & 3.274 & 7.002 & 113,8 \\
Madrid & 1.189 & 3.552 & 198,7 \\
Murcia & 1.376 & 2.735 & 98,7 \\
Navarra & 2.592 & 4.017 & 54,9 \\
País Vasco & 1.785 & 3.721 & 108,4 \\
La Rioja & 441 & 987 & 123,8 \\
\hline Total & 46.355 & 136.704 & 194,9 \\
\hline
\end{tabular}

FUENTE: INE, Encuesta de ocupación en alojamientos de turismo rural, 2001-2010

Con el objetivo de unificar los datos, ofrecemos las estimaciones que recoge el INE tomando como mes de referencia agosto, que es cuando hay un mayor volumen de establecimientos abiertos. Hubiese sido interesante retrotraernos más en el tiempo para poder apreciar todavía con mayor nitidez la rápida ascensión de este tipo de alojamientos. Como se puede observar, el crecimiento ha sido muy importante en todas las CCAA, duplicándose como mínimo el número de plazas. En general sigue significando una parte reducida de la oferta total, con la excepción de algunas comunidades autónomas como Castilla y León en donde representa más del $20 \%$ de las plazas y sobre dos tercios de los establecimientos. En conjunto no se puede negar el éxito que ha tenido, al menos en términos cuantitativos, gracias al incremento de una oferta que hace apenas unas décadas no existía. Sin embargo, transcurrido ya algún tiempo desde que empezó el interés por su desarrollo, es bueno hacer balance y reflexionar sobre su evolución y sus perspectivas, toda vez que los acelerados procesos de cambio en nuestra sociedad también afectan de forma muy clara a todo el sistema turístico. Nos referimos, por ejemplo, al tiempo libre cada vez más fragmentado, a la mayor movilidad de las personas o a la irrupción de las compañías aéreas de bajo coste. 


\section{DEL TURISMO DE INTERIOR A LOS TURISMOS DE INTE- RIOR}

Aunque ya hace años que se habla de turismos de interior (VALENZUELA, 1997), lo cierto es que el uso del plural parece ahora más pertinente que nunca. Es verdad que, aún bajo el dominio aplastante del sol y playa, siempre se mantuvo una oferta a veces de carácter masivo, como las estaciones de esquí, a veces de elevada frecuentación, como muchas ciudades y pueblos, y a veces de carácter mucho más restrictivo, como los espacios naturales y los rurales. Sin embargo en los últimos tiempos no solo ha aumentado esta diversificación sino que también se ha intensificado y visibilizado. De esta forma la oferta interior es hoy extraordinariamente rica y, en cierta medida, cada vez más relacionada con la propia del litoral. De hecho estas últimas áreas incorporan de forma creciente el post-país con sus valores rurales y naturales como un atractivo más que enriquece una oferta que se mueve en ámbitos cada vez más competitivos.

En el caso concreto de Galicia, el título de este apartado tiene, si cabe, más significado en la medida en la que la oferta existente hasta la década de 1980 era casi exclusivamente de litoral, con alguna excepción. Sin considerar la ciudad de Santiago de Compostela, el panorama era bastante pobre: una pequeña estación de esquí, algunos balnearios con clientela básicamente subvencionada a través de programas públicos de salud; desinterés urbano por el turismo en las capitales de interior; y espacios naturales protegidos muy escasos y sin apenas gestión. En definitiva, la oferta de alojamiento se reducía a algunos campings, hoteles y otras fórmulas en general de perfil bajo, además de una significativa ausencia de producto.

Sin embargo a lo largo de la década de 1990 y de la siguiente, se fueron experimentando cambios muy significativos. Como ya es conocido, el despegue del turismo en Galicia se produce en torno al año santo de 1993, si bien desde finales del decenio anterior se venía observando una creciente atención sobre este fenómeno. Aunque es conveniente no olvidar que la oferta de litoral también se reforzó considerablemente, en las políticas públicas se dio mayor visibilidad tanto al turismo rural como a los caminos de Santiago, que transcurrían siempre por itinerarios interiores, con alguna excepción.

En relación a los caminos de Santiago se hizo un gran esfuerzo público en su delimitación y en la apertura de albergues para acoger a los peregrinos. Este último hecho sin duda condicionó el resto de la oferta de alojamiento. Con todo es muy significativo el incremento de establecimientos que se produce a partir del Xacobeo 93. En un informe realizado por la Universidad de Santiago de Compostela (USC) en el año 2008 en relación al alojamiento en 
el Camino Francés, con mucho el más frecuentado, se daba cuenta que en su tramo gallego había 79 establecimientos de los que solo el $20 \%$ eran hoteles, y el resto hostales y pensiones hasta cubrir un total de algo más de 1.100 plazas. De ese número, más de la mitad abrieron a partir de 1994 y fundamentalmente desde el año 2000. Además, prácticamente la totalidad de estos nuevos locales se localizan a pie del camino o, en todo caso, a menos de 1 $\mathrm{km}$ del mismo. Y un dato más de interés está en el hecho de que los gerentes señalan que de media un $75 \%$ de la clientela son peregrinos.

Este mismo informe dedica un apartado a las casas de turismo rural, cuyo número asciende a 44 para algo más de 300 plazas. En este caso tenemos también una estrecha relación con el Camino, puesto que el $44 \%$ de los establecimientos se encuentran a pie de ruta y un $20 \%$ a menos de un kilómetro. Además el $92 \%$ de los mismos se inauguraron en fecha posterior a 1993. En lo relacionado con el tipo de clientela, se advierte que en aquellas casas más próximas al Camino el porcentaje de peregrinos supera el $80 \%$, descendiendo progresivamente hasta el $35 \%$. Sin embargo no es una reducción lineal puesto que hay casas más alejadas de la ruta que tienen valores muy elevados de ocupación de peregrinos debido fundamentalmente a promociones y servicios específicos para este segmento.

Por lo tanto podemos ver una relación muy estrecha entre el Camino de Santiago y la multiplicación de la oferta de alojamiento interior, sobre todo a partir del año 1993 que es cuando realmente se empieza a ver la dimensión del fenómeno jacobeo lo que estimula la toma de iniciativas empresariales en las inmediaciones de la ruta. Las ayudas públicas procedentes de diversas administraciones, desde la europea hasta la autonómica, tuvieron un protagonismo importante para la financiación de buena parte de los nuevos equipamientos. Incluso se puede decir que algunas iniciativas y programas le dieron un excesivo peso al turismo, en algunas ocasiones de forma indirecta a través por ejemplo de la recuperación de patrimonio material.

Además de los caminos de Santiago, el turismo rural fue el auténtico protagonista de la política turística gallega durante la década de 1990 y parte de la siguiente, aunque en ocasiones las dos ofertas iban muy estrechamente relacionadas. La legislación que regula esta tipología es del año 1995. En ella, entre otros asuntos, se fija una clasificación de los establecimientos en tres grupos, A, B y C, que en buena medida responden a los mismos criterios que se utilizaban en Portugal en donde la norma era de una década atrás, de 1984. ABAD (2003) nos explica muy bien el desarrollo en Galicia de este subsector. La nueva Ley de Turismo de Galicia, aprobada definitivamente en 2011, insiste en singularizar estos alojamientos, si bien introduce algunas novedades. 
El crecimiento de la oferta de alojamiento vinculada con el turismo rural experimentó un desarrollo muy importante. Si en términos absolutos su volumen total no era especialmente significativo para el conjunto de Galicia, en valores relativos son cifras que llaman la atención. Por ejemplo, si consideramos 1995 como referencia 100 en número de plazas, en el año 2000 se llega al punto 246, en tanto que los hoteles, también en fuerte expansión, alcanzan el 125. Si esto lo traducimos en números estamos hablando de 129 establecimientos y 1.262 plazas en 1995 , que son 283 y 3.108 en 2000, continuando en los años posteriores esta misma tendencia, y a veces incluso acelerándose.

Como señalábamos antes, la financiación pública fue fundamental para entender este crecimiento. De hecho los programas de desarrollo rural, que buscaban mantener población y actividad económica sin incrementar la producción agrícola, encontraron en el turismo una buena oportunidad para aplicar esta política. SPARRER (2007) recoge algunas cifras muy representativas de esta realidad. Por ejemplo la medida 3 del Leader I (1991-94) referida a turismo rural acaparó el 63,6\% de la inversión en Galicia y en alguna comarca, como en Portodemouros, llegó al 70\%. En el Leader II (1994-99) la medida B3 siguió siendo la más voluminosa con el $31 \%$ de la inversión, superando el $50 \%$ en ciertos espacios. Otros autores como PÉREZ FrA y LÓPEZ IGLESIAS (2005) evidencian desde un punto de vista crítico esta misma circunstancia. También en el programa Proder nos encontramos con situaciones similares, como señalan CÀNOVES et AL. (2006). Por lo tanto la expansión del turismo rural no se puede vincular solo, ni tan siquiera principalmente, a iniciativas sectoriales si no que responde a una estrategia más amplia relacionada con la ordenación del territorio (LOIS y SANTOS, 2004; RODRÍGUEZ y PÉREZ, 2004) en un ámbito acuciado por problemas de abandono, de pérdida de identidad y de grave deterioro socio-económico (MARTÍNEZ, 2004).

A todos estos programas, iniciativas y ayudas que estimularon el turismo de interior en Galicia se le añaden los Planes de Dinamización Turística (PDT) que, lanzados desde la correspondiente Secretaría de Estado de Comercio y Turismo, sirvieron para proyectar destinos emergentes. Puestos en marcha en 1996, en el caso de Galicia el primer territorio beneficiado fue la montañosa comarca de Trives (Ourense) que inicia sus trabajos en el año 1997. A partir de 2001 otras áreas del interior de Galicia se verán beneficiadas por las actuaciones derivadas de los PDTs.

En definitiva, llegados al inicio del siglo XXI nos encontramos con un panorama turístico que había cambiado bastante rápidamente en apenas una década y que básicamente seguirá la misma línea en los años siguientes. El 
turismo de interior comienza a ser una opción interesante para muchos turistas, no solo el Camino de Santiago, sino también la oferta de alojamiento rural en sentido estricto. Sin embargo, a medida que avanza el nuevo siglo, se producen otras mudanzas que vuelven a dibujar un escenario cambiante y muy sugerente.

Entre los cambios más importantes que podemos señalar, en primer lugar habría que hacer referencia a la incorporación de los ámbitos urbanos como atractivos turísticos. No nos referimos tanto a ciudades como A Coruña o Santiago, sino a otras hasta el momento ajenas al fenómeno turístico, e incluso villas del interior de la Comunidad Autónoma. En cuanto a estas últimas, los antecedentes están en Allariz (Ourense). Este pequeño núcleo comenzó una profunda transformación a principios de la década de 1990 que, entre otras cosas, supuso una importante recuperación de su patrimonio arquitectónico y natural, yendo en dirección contraria a la tendencia dominante en la mayor parte de las villas gallegas: la de estimular el crecimiento urbano a base de una expansión constructiva descontrolada que acabó por desfigurar muchos de estos espacios. El reconocimiento a la labor realizada en Allariz fue de carácter internacional con el Premio Europeo de Urbanismo en 1994 o la declaración de Reserva de la Biosfera, entre otros.

Lo más relevante del caso que estamos comentando es que, sin ser el de Allariz un programa estrictamente turístico, tuvo unas consecuencias evidentes sobre el desarrollo de este sector. De hecho nos parece significativo indicar que si en 1995 solo había 2 casas de turismo rural y 4 pequeños establecimientos con un total de unas 75 plazas, en la actualidad son 5 las casas de turismo rural, un camping de más de 200 plazas y varios hoteles y pensiones más, incluida la presencia de cadenas hoteleras. A partir de este ejemplo pionero, el turismo comenzó a entrar en la agenda de muchos núcleos urbanos pequeños del interior, apoyados a veces por programas en destino como aconteció con el citado PDT de Trives o el de Ribadavia (Terras do Avia).

Pero no fueron solo las villas, sino también las ciudades. El ejemplo más evidente fue el de Lugo, cuyo PET en cierta medida avanzó el creciente interés de las ciudades por el turismo, algo que con la excepción de Santiago y A Coruña, apenas había existido hasta el momento. De hecho, para la capital lucense la falta de referencias llevó a tratar de desarrollar un modelo claramente inspirado en la exitosa trayectoria de Compostela.

De vuelta al mundo rural, la diversificación de la oferta turística avanzó significativamente por dos vías: la termal y la de hoteles monumento. Sin embargo los espacios naturales no fueron objeto de tanta atención, por lo menos de forma directa, debido en algunos casos a la incapacidad para ges- 
tionar áreas de enorme atractivo, como las reservas de la biosfera, pero también por la singular carencia de espacios protegidos, fundamentalmente de parques nacionales. De hecho, el único existente es el de las Islas Atlánticas. En su estudio sobre el uso público de los espacios naturales gallegos, CABALAR (2010) se refiere al desigual uso turístico de estos entornos contrastando la fuerte presión que sufren aquellos próximos a áreas litorales frente a aquellos otros localizados en comarcas interiores.

En cuanto a la oferta termal es cierto que la misma no es estrictamente de interior, si no véase el caso de A Toxa, pero la localización de las fuentes minero-medicinales coincide principalmente con áreas del sur de la Comunidad, fundamentalmente de Ourense. La extraordinaria riqueza que posee Galicia es aprovechada con fines turísticos desde el siglo XIX, como lo demuestran los magníficos ejemplos de Mondariz o A Toxa, entre otros muchos. Sin embargo, la crisis, que se acentuó en la segunda mitad del siglo $\mathrm{XX}$ en el mejor de los casos, llevó al cierre de muchos balnearios, mientras que otros se transformaron en centros de salud vinculados a programas sociales para población de la tercera edad, siendo en general poco atractivos para la mayor parte de los turistas.

Sin embargo, a mediados de la década de 1990 comienza una recuperación significativa del termalismo que se va a acelerar sobre todo a partir del comienzo del nuevo siglo. Como antecedentes inmediatos DEL MORAL (s.f.) cita los estudios encargados por la Secretaría Xeral para o Turismo de la Xunta de Galicia: Estudio para el desarrollo del turismo termal en Galicia (1991) y el Estudio de intervenciones a realizar en 23 instalaciones termales de Galicia para su adecuación a las exigencias actuales (1995). Estos y otros trabajos más ayudarían a redactar y aprobar la Ley 5/1995 de regulación de las aguas termales, de manantial y de los establecimientos balnearios de la Comunidad Autónoma de Galicia, así como el Decreto 402/1996 que regula y ordena el sector (DEL MORAL, s.f.).

El establecimiento pionero en este proceso fue el de Mondariz, sin duda el más importante por su trayectoria histórica. Aunque en tierras interiores, se localiza muy cerca de las Rías Baixas y de mercados tan potentes como el de Vigo. Pero por esas fechas aparecen otras iniciativas que son las que realmente van a marcar el renacer del termalismo. Como ejemplos señalamos el Balneario de Arnoia (Ourense), de nueva concepción y que será el buque insignia que colocará a Ourense, una provincia de escasa tradición turística, en posición de liderazgo termal en España; un segundo ejemplo es el de Guitiriz (Lugo),que ya iniciado el siglo XXI renace de sus viejas instalaciones en medio de una de las comarcas más ganaderas de Galicia. 
Como decíamos, una vez que se inicia el siglo XXI, se observa un continuo goteo de nuevos balnearios, algunos recuperando su historia y otros no, tan solo aprovechando la existencia de aguas termales y una demanda creciente por parte de una clientela diferente a la que ya parecía habitual. También en esos años se empieza a celebrar Termatalia, una Feria Internacional que cada año tiene lugar en Ourense y que es la referencia para todo el sector. Según los datos emanados de esta organización, Galicia cuenta con unas 3.000 plazas en 20 balnearios y 2 centros de talasoterapia, es decir que representa aproximadamente el $20 \%$ de la oferta española en este segmento (www.termatalia.com).

Otra de las vías que citamos en relación a la diversificación de la oferta de alojamiento en el mundo rural vino de la mano de los hoteles monumento. Este concepto deriva del aprovechamiento que se le va a dar a importantes edificios localizados en el mundo rural, y que son sobre todo antiguos monasterios, muchos de ellos abandonados. En este caso ejerció de pionero el monasterio de San Clodio (Ourense). Plantado en la comarca vitivinícola del Ribeiro, este edificio comenzó en el siglo XII albergando monjes del Císter. Su completa reforma para convertirlo en hotel en el año 2000 representa un hito para actuaciones posteriores. En efecto, en los años siguientes se multiplican las inauguraciones siendo el ejemplo más significativo de esta modalidad de establecimientos el Parador de Santo Estevo de Ribas de Sil, en la Ribeira Sacra de Ourense, abierto en el verano de 2004.

\section{SITUACIÓN ACTUAL}

Hasta ahora hemos estado haciendo un breve recorrido por la historia más reciente de los turismos de interior de Galicia. Se observa efectivamente la importancia creciente que van adquiriendo nuevas tipologías de alojamiento. Sin embargo, a pesar de esta diversificación, la oferta litoral sigue siendo claramente dominante, además de la urbana gracias al peso que tiene la ciudad de Santiago, aunque también en los últimos años A Coruña y Vigo han conseguido hacer frente al déficit cuantitativo y cualitativo que tenían en lo referente a plazas hoteleras.

De hecho, si nos fijamos en la distribución espacial de la oferta de alojamiento en Galicia, observamos que las provincias de A Coruña y Pontevedra concentran cerca del $80 \%$ de la misma, en tanto que Ourense no llega ni siquiera al $8 \%$. Se manifiesta una incapacidad a lo largo del tiempo para reducir esa enorme diferencia. Municipios litorales como Sanxenxo, O Grove, Poio, todos en la provincia de Pontevedra, además de otros urbanos, como Santiago o A Coruña, acaparan un volumen muy significativo del total de camas turísticas de Galicia. Incluso en el turismo rural se observa un fenó- 
meno que no deja de ser contradictorio: el reparto de plazas reproduce el mismo orden que el de la oferta global, de tal forma que Pontevedra y A Coruña ocupan los primeros puestos y Ourense el último.

Es cierto que una visión más detallada nos hace ver que buena parte de los establecimientos se dispersan por comarcas interiores, pero no deja de ser interesante comprobar cómo hay un volumen significativo que se localiza cerca del mar. Esto ratifica la hipótesis que M. SPARRER (2007) desarrolló en su tesis doctoral, según la cual muchos alojamientos de turismo rural sirven sobre todo de plataforma para hacer actividades vinculadas más con productos de sol y playa o urbanos. Esta misma idea aparece recogida por GRANDE (2010) cuando expone que, a pesar de que la naturaleza y el paisaje son los elementos que más gustan a los clientes alojados en establecimientos rurales de Ávila, según la percepción de los propietarios, resulta que la visita a la ciudad de Ávila es la actividad más frecuente de los clientes.

El mapa que presentamos refleja de manera gráfica lo que estamos comentando. Una gran dispersión territorial aunque con tendencia a la densificación en ciertos territorios. Las principales concentraciones las observamos en la provincia de Pontevedra y en el Ssur de A Coruña, muchas veces coincidiendo con sectores costeros o en los entornos de las principales ciudades. Es verdad que también se aprecian volúmenes significativos en tierras interiores, como es el caso del Este de Lugo, Nordeste de Pontevedra, Sureste de A Coruña y noroeste de Ourense. Sin embargo, es precisamente en alguna de las comarcas más rurales y montañosas donde se encuentran los principales vacíos, como son los ejemplos de la penillanura de A Coruña, las áreas ganaderas del centro de Lugo o el macizo central ourensano.

En cualquier caso, a parte de esta desigual distribución espacial, nos interesa destacar otras cuestiones relacionadas con la situación actual del turismo rural. La primera es que el número de establecimientos se sitúa cerca de los 600 para un total de 7.000 plazas, lo que se corresponde con el 21 y el $6 \%$ respectivamente de la oferta global de Galicia. Este diferente peso de alojamientos y plazas obviamente reside en que el tamaño de los establecimientos es bastante reducido, en parte debido a la propia normativa, en relación a las otras tipologías, sobre todo hoteles y campings. También es relevante indicar que en los últimos años se observa un crecimiento muy pausado en comparación con los periodos anteriores, sin duda porque desde la administración autonómica se considera que la situación actual es suficiente, y solo se plantearía el apoyo en caso de no existir oferta alguna en el municipio, o para la tipología $\mathrm{D}$, de aldeas rurales, que fue introducida en la norma con la reforma de 2004. Una segunda cuestión a comentar tiene que ver con una elevada dispersión del alojamiento, de tal forma que aproximada- 
mente dos tercios de los 315 ayuntamientos gallegos tienen al menos 1 casa, incluyendo la práctica totalidad de los de la provincia de Pontevedra; sólo en Ourense se advierte un vacío en algo más de la mitad de los municipios.

Mapa 1. Distribución de los establecimientos de turismo rural.

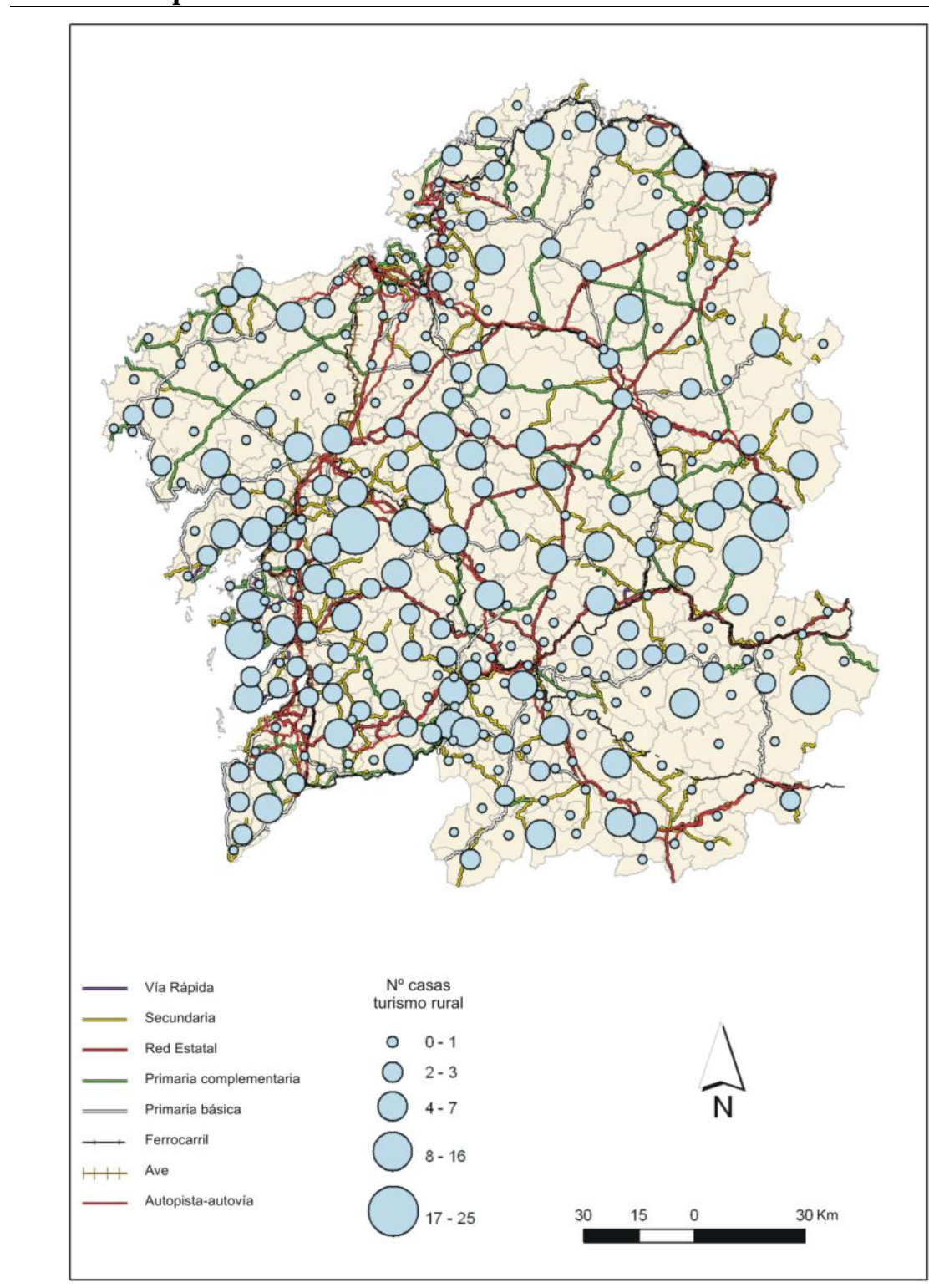

FUENTE: Sobre la base de LOIS, PIÑEIRA y SANTOMIL (2009) 
En los últimos años el turismo rural en Galicia ha dejado claramente de ser una prioridad en las políticas públicas, al menos tal y como se entendía. Como ya fue comentado, salvo casos excepcionales, no se dan ayudas públicas para la apertura de nuevos establecimientos. Las preocupantes tasas de ocupación nos dejan ver con toda claridad la inadecuación entre la oferta y la demanda. Según los datos del INE, la ocupación por habitación en 2010 fue del $16 \%$, dos puntos por debajo de la media española, incluso considerando que fue año jubilar. Es más, se aprecia en los últimos años un ligero retroceso, situación muy delicada teniendo en cuenta que se partía ya de valores muy bajos de ocupación. Además, el hecho de que el grado de ocupación sea significativamente más alto en el litoral (22\% en las Rías Baixas), no hace más que confirmar la hipótesis citada anteriormente del trabajo de $\mathrm{M}$. SPARRER (2007).

Evidentemente, estos últimos datos tienen que servir de reflexión sobre cuáles son las causas que explican estas cifras. Independientemente de que puedan existir ocultamientos, tal vez habría que darle importancia a aspectos tales como: abundancia de alojamiento frente a una alarmante escasez de producto; competencia de otras ofertas en el ámbito rural; y aparición de destinos urbanos de gran atractivo a precios muy económicos gracias a las compañías aéreas de bajo coste.

En relación a la ausencia de producto, existe una clara conciencia del mismo por parte de la administración autónomica. De hecho se han realizado numerosos esfuerzos por ampliar y mejorar la oferta complementaria. Podemos destacar el trabajo que se hace con el diseño de las rutas del vino o con la riqueza gastronómica. En cuanto a paquetes, tal vez lo más interesante haya sido la creación de Bono Iacobus, que generó una red de alojamiento rural para peregrinos incluyendo una serie de facilidades que lo hacen muy competitivo y atractivo. También existe el programa Vive Galicia, que empaqueta a la carta experiencias, incluidos destinos rurales. De interés resulta igualmente Xuventur, que intenta acercar a los jóvenes gallegos el turismo de aventura en el medio rural gallego. O, por último, el Otoño gastronómico, que en este caso une de nuevo los establecimientos rurales con la gastronomía de temporada y cuyos resultados en 2010 se incrementaron un $20 \%$ respecto a 2009.

De todas formas, como hemos visto, no parece que el problema sea en exclusiva la falta de producto. Los datos apuntan a que, a pesar de todo, continúa la caída en las tasas de ocupación así como la propia estancia media. Esto nos puede llevar a pensar en la escasa rentabilidad económica de muchos de los negocios. Si consideramos que desde 1995 los establecimientos subvencionados tenían que permanecer en el programa al menos 15 años, esta- 
mos llegando al período límite para que muchas de esas casas puedan optar a salir del mercado turístico. Esta es una posibilidad muy real, sobre todo si consideramos el alto porcentaje de viviendas subvencionadas: ABAD (2003) estimaba que a finales del año 2000 el $64 \%$ del total de plazas ofertadas en la provincia de Ourense habían recibido algún tipo de ayuda de la Xunta de Galicia. Esta autora estima que, si incluimos otras subvenciones, en algunos años se llegaría al 100\% de plazas subvencionadas.

Volviendo a la situación actual del turismo rural, decíamos que sin duda tienen que existir otros motivos más allá de la falta de producto que expliquen los bajos índices de ocupación, a no ser que esta nueva oferta que se creó en estos últimos años no sea lo suficientemente potente como para atraer a más clientes. La competencia directa de otro tipo de oferta mayoritariamente anclada en el ámbito rural puede ser un motivo más que ayude a entender esta falta de fortaleza del turismo rural. Nos referimos por ejemplo al termalismo que ya vimos como tuvo un crecimiento muy importante con la apertura de nuevos establecimientos. Los datos que hemos podido encontrar en fuentes como Hosteltur, procedentes de la propia Asociación de Balnearios de Galicia, hablaban para principios del S. XXI de unas tasas de ocupación media del $80 \%$, bastante coincidentes con los que cita DEL MORAL (s.f.), y que la AETEGAL (Agrupación Empresarial Innovadora Termal de Galicia) sitúa ya al final de la primera década en el $70 \%$. Evidentemente, aunque una parte significativa de la clientela se acoge a programas públicos subvencionados, no se puede negar el éxito creciente de esta oferta entre personas urbanas de edad media. ALÉN y FRAIZ (2006) destacan como perfil socio-económico de los usuarios de balnearios de Galicia su procedencia mayoritariamente española (y sobre todo gallega), predominio de parejas, más mujeres que hombres, trabajando por cuenta ajena y funcionarios, con estudios superiores, comprendidos fundamentalmente en el grupo de edad entre 35 y 54 años, y con ingresos medios mensuales medios o medio/altos. De todas formas, como hemos comentado, estos resultados de ocupación están en buena parte relacionados con el hecho de que Galicia es una de las CCAA que más plazas y más balnearios aporta al programa de Termalismo Social del IMSERSO.

Además del termalismo, hay otra oferta que va adquiriendo mayor protagonismo en estos últimos años y que se localiza muy mayoritariamente en tierras interiores. Son los hoteles monumento. En estos momentos, y según figura en la página web de Turgalicia, existen 17 establecimientos dentro de esta categoría. Aunque hay algunos que llevan muchos años abiertos, éstos se encuentran preferentemente en zonas urbanas y de costa. Pero a nosotros nos interesan sobre todo los que se localizan en el interior y cuya vida em- 
presarial todavía es muy corta. Como ya hemos comentado el pionero fue el monasterio de San Clodio, en la comarca ourensana del Ribeiro, si bien el ejemplo más espectacular es el monasterio de Santo Estevo de Ribas de Sil (Ourense), que pertenece a la red de Paradores de España. A ellos habría que unirles edificios históricos como el castillo de Maceda (Ourense), el Palacio de Sober (Lugo) o el Pazo de Lestrove (A Coruña), entre otros.

Se trata de un concepto no exclusivamente rural pero que en realidad, cuando se instala en estos ámbitos, hace una competencia directa a los establecimientos de turismo rural, e incluso al termalismo. La categoría de aquéllos se basa, como ya vimos, en la antigüedad y calidad del edificio, ocupando el Grupo A fundamentalmente los pazos. Ahora se da un paso más al unirse a ellos algunos de los mejores pazos así como antiguos monasterios. La gestión de estos negocios a menudo pertenece a grupos empresariales o a cadenas hoteleras con todo lo que eso supone en términos de comercialización, asociando a la espectacularidad del alojamiento y, a menudo, del emplazamiento, unas instalaciones que no tienen las limitaciones del turismo rural en cuanto al número de habitaciones, lo que permite realizar además inversiones propias de hoteles vacacionales. Entre estas últimas destacan los spas que permiten presentar una oferta concurrente con la de los balnearios al menos en el uso del agua, que no en sus propiedades.

Esta tendencia a la monumentalidad del alojamiento rural se desplaza también a pequeños núcleos urbanos como Monforte de Lemos en Lugo. Se trata de un comportamiento que debería comenzar a llamar la atención en relación al clásico turismo rural. Esta afirmación se basa en el hecho de que en los últimos años muchas villas del interior han empezado a demostrar interés por el turismo como respuesta a la crisis económica y demográfica que también les ha afectado a ellas. Aprovechando muchas veces la ausencia de actividades en el mundo rural, las villas se intentan posicionar como capitales de esos territorios ofreciendo no solo la proximidad a esos ámbitos, sino también su patrimonio y su historia, además de múltiples servicios. Esto lleva a que, por ejemplo, la Ribera Sacra poco a poco vea desplazado su centro de gravedad desde unas áreas naturales y culturales a los espacios urbanos del entorno. GRANDE (2010) verifica esta tendencia en La Rioja al demostrar que la mayoría de los visitantes a la comarca vitivinícola de La Sonsierra pernoctaban en las ciudades próximas, sobre todo Logroño y Haro; igualmente cita la estrategia de posicionamiento turístico de la localidad de Calahorra que se sustenta en La Ciudad de la Verdura. Este último comportamiento es fácil de observar en muchas otras villas que buscan centralizar las riquezas de sus respectivas áreas rurales. Tal vez habría que comenzar a in- 
vertir más esfuerzos en la creación de sinergias entre destinos de ciudad y rurales, especialmente en relación a los pequeños núcleos urbanos.

En otro orden de cosas no debemos de olvidar que también en los años más recientes ha surgido una fuerte competencia por parte de destinos urbanos. No es sólo que las villas y ciudades gallegas, como ya hemos comentado, muestren un creciente interés por captar visitantes. Se trata también de la aparición de las líneas aéreas de bajo coste, que además de traer turistas incorporan una oferta atractiva de destinos españoles e internacionales. No existe ningún estudio, al menos en Galicia, que vincule la relación entre las compañías aéreas de low cost con la debilidad del turismo rural. Aunque es cierto que ésta es persistente desde su creación, ya hemos visto que parece que se agudiza con el tiempo a pesar de los esfuerzos en la creación de producto. Lo que nos lleva a determinar esta relación está en el hecho de que, de forma genérica, los perfiles de los clientes de turismo rural y urbano son similares: parejas o en general gente joven, clase media y niveles de formación elevados. Si a esto le añadimos la tan repetida idea de que el turista contemporáneo busca experiencias, actividades y que se caracteriza por una explosión de motivaciones, podremos entender que no sería extraña una transferencia desde el turismo rural clásico hacia otras ofertas más atractivas en el mismo ámbito y sobre todo hacia los espacios urbanos. Este cambio tan radical, probablemente difícil de entender en otro momento, hoy solo evidencia que estamos delante de un turista contemporáneo.

También habría que considerar los efectos de la crisis económica, muy agresiva desde el segundo semestre del 2008. Los cambios en el comportamiento de los turistas apuntan hacia una disminución del número de desplazamientos, con una concentración en el viaje principal y una recuperación de los paquetes. Esto significa que los destinos menos perjudicados son los de sol y playa. Dentro de lo que fue el mal año 2009, el turismo urbano, al menos a nivel europeo y mundial, fue uno de los más perjudicados, precisamente por esos movimientos de adaptación a la crisis. Aunque los organismos oficiales no hacen demasiado caso al turismo rural debido a su menor importancia, es fácil pensar que su trayectoria también se haya visto influenciada por estos acontecimientos, acrecentándose a causa de una atractividad menor.

Muy en relación con el turismo rural están los espacios naturales protegidos sobre los que poco se ha avanzado en relación a su catalogación y gestión. Por ejemplo, áreas de enorme interés ambiental y cultural como los Ancares y el Courel tienen en la práctica unos niveles de protección muy bajos a pesar de estar incluidos en la Red Natura. Por su parte las 5 Reservas de la Biosfera de Galicia poseen un título que hasta el momento es fundamental- 
mente nominativo y poco ejecutivo. En realidad a las áreas naturales protegidas de Galicia le pasa un poco como al turismo rural en cuanto a su vinculación estrecha con la oferta litoral. El único parque nacional es el de las Islas Atlánticas. El número de visitas de los dos principales conjuntos, Ons y Cíes, se estima en más de doscientos mil a través de las navieras, más otras veinticinco mil personas en embarcaciones privadas (CABALAR, 2010); y del total aproximadamente un $75 \%$ se concentra en julio y agosto. Estamos por lo tanto hablando de una motivación básica de sol y playa que secundariamente se combina con elementos propios del turismo de naturaleza. El caso del parque natural del complejo dunar de Corrubedo y lagunas de Carregal y Vixán no es muy diferente del anterior, según las informaciones que nos aporta CABALAR (2010), aunque al no ser insular la presión es aún mayor: afluencias estivales de junio a septiembre en torno a las trescientas cincuenta mil personas.

Además de estos dos espacios naturales protegidos de litoral, hay en Galicia otros dos próximos a la costa, cercanos a importantes focos emisores, y que también sufren una importante presión no siempre vinculada a sus valores ambientales. El primero de ellos es el parque natural del monte Aloia en la localidad fronteriza de Tui, que tiene una vocación fundamentalmente recreativa (CABALAR, 2010). Recibe anualmente unas veinte mil visitas, concentradas principalmente en el verano si bien la importancia que tienen los grupos de escolares, por motivos educativos, hace que también la primavera tenga una afluencia significativa.

En el norte de la Comunidad Autónoma nos encontramos con otro parque natural, el de las Fragas del Eume, que también está muy próximo a áreas litorales de gran presión urbana y turística. Al contrario que el anterior, sus valores ambientales dominan sobre los puramente recreativos. Su creciente grado de conocimiento ha incrementando sustancialmente el número de visitas que se va aproximando a las cincuenta mil, de las que cerca del 50\% tienen lugar en julio y agosto. Es significativo que, según recoge CABALAR (2010) en 2008, menos del $10 \%$ del total de visitantes fue al centro de interpretación. Los datos que nos aporta este autor, procedentes de las memorias del propio parque, nos indican que más de la mitad de los usuarios son de origen gallego y que la motivación disfrutar de la naturaleza es citada sólo por el $12 \%$ de los encuestados.

Por último vamos a hacer alusión a tres parques naturales interiores bien distintos a los que hemos citado hasta el momento. En primer lugar está el del Invernadeiro en Ourense, con acceso restringido y una cuota máxima de visitantes diarios. Este hecho no deja de ser paradójico si consideramos que las tres mil registradas suponen apenas el $10 \%$ del total de las permitidas 
(PAÜL, 2009). En los límites con la provincia de León, en la comarca ourensana de Valdeorras, está el parque de la sierra de Enciña da Lastra con poco más de mil visitas al año de origen fundamentalmente comarcal. Finalmente una breve alusión al parque de Baixa Limia-Xurés que da continuidad al portugués de Peneda-Gerês. El volumen de visitas oscila entre cinco y seis mil y el motivo principal está en el balneario, el rio y el baño (CABALAR, 2010).

En definitiva resulta complicado relacionar la visita a los espacios naturales protegidos de Galicia con el turismo de naturaleza. Los datos que nos aportan autores como CABALAR (2010) más bien sugieren que la playa, la recreación y en todo caso la educación ambiental son los dominantes, si bien admitimos que siempre los paisajes y los valores naturales tienen un significado protagonismo. Sin duda la oferta de turismo rural debería comenzar a incorporar de manera más evidente estas áreas, cuyo potencial es indiscutible. De hecho en las encuestas a la demanda aparece de manera reiterada como primera motivación de la visita a Galicia: Conocer el medio natural y los paisajes.

Pero sin duda la gran joya del turismo de interior de Galicia, al menos en términos de producto, es el Camino de Santiago. Sobre el mismo pivota una parte de la oferta de establecimientos rurales y algunos de los paquetes de mayor éxito, como el Bono Iacobus. La cuestión más paradójica surge de la comparación entre el extraordinario crecimiento de peregrinos y los limitados efectos positivos que genera en los territorios que atraviesa, sobre todo en la ruta francesa, con mucho la más frecuentada. Son varias las causas que nos permiten entender esta situación. En primer lugar, la existencia de una red de albergues públicos, y en menor medida privados, que reducen significativamente el uso de alojamientos hoteleros. En segundo lugar, están las características propias del viajero, incluidas las motivaciones principales, que le llevan a valorar el Camino en sí mismo y a mantener un comportamiento más austero en relación al gasto y al consumo del entorno. Esto quiere decir que, además de reducir las compras de recuerdos por razones obvias de peso, la alimentación acostumbra a producirse en ambientes dominados por los propios peregrinos, como los albergues o los restaurantes con menús específicos; además el destino es el Camino que lleva a Santiago o a Finisterre, de ahí el deseo de ir siempre avanzando, independientemente de que no se puede pernoctar dos veces seguidas en el mismo albergue sin que haya causas de fuerza mayor, como una enfermedad.

Las consecuencias de todo lo anterior es que el peregrino gasta muy poco dinero, entre 25 y 30 euros por jornada, y apenas se aparta de lo que es estrictamente la senda que le lleva hasta Santiago. Tal vez sería conveniente comenzar a repensar algunos aspectos vinculados con estas vías de peregri- 
nación. Recordemos que en su momento el reconocimiento de las diferentes rutas fue defendido como fórmula para generar desarrollo en todos estos territorios de interior. Por lo tanto habría que retomar ese discurso y plantearse la creación de nuevos escenarios en los que, sin perder ni los atractivos ni la esencia del Camino, se propongan medidas y acciones que aporten dinamismo y riqueza, no solo a puntos concretos, sino también a todo el entorno de los diferentes caminos. El largo periodo de 11 años que se abre entre los jacobeos de 2010 y 2021 ofrece una buena oportunidad para la experimentación y la innovación con el objetivo de maximizar los beneficios que se derivan de un producto turístico de éxito que atraviesa comarcas interiores que siguen, a pesar de todo, inmersas en una profunda crisis.

\section{CONCLUSIONES Y REFLEXIONES PARA EL FUTURO}

Es difícil hacer unas generalizaciones sobre el futuro de los turismos de interior en Galicia debido a la diversificación de la oferta existente. Sin embargo, creo que es posible afirmar que existe una gran incertidumbre sobre lo que acontecerá en los próximos años. A crear esta situación ayuda, no sólo la crisis económica, sino también diferentes elementos que se vienen arrastrando. Entre ellos está la agonía y atonía en la que sobreviven los espacios rurales gallegos, y también la mayoría de los españoles, que contribuye a restar atractivo a una actividad que se caracteriza precisamente por su gran dinamismo; o en todo caso sirve para transformar el turismo rural en turismo natural como consecuencia del abandono de los campos. No debemos olvidarnos tampoco de que muchas de las iniciativas surgen desde arriba, lo que deriva en una escasa implicación y participación de las poblaciones locales. $\mathrm{La}$ idea del turismo como agente inductor del desarrollo rural parece cada vez alejada de la realidad, incluso diría que se aparta del discurso político. Como señalan GIL et AL. (2010), es necesario incorporar nuevos enfoques vinculados a conceptos como valor añadido, diferenciación, innovación y creatividad, correspondiéndole al patrimonio inmaterial un mayor protagonismo. Es cierto que se están haciendo importantes esfuerzos en crear producto, en formación, o en destacar aspectos vinculados con las experiencias. Sin embargo los resultados medidos en tasas de ocupación no dejan de disminuir año tras año.

El termalismo, que parece que desplaza con fuerza al turismo rural en las preferencias tanto de la oferta como de la demanda, tampoco creemos que vaya a contribuir de manera notable a ese desarrollo. Más allá de la creación de empleo, cuestión sin duda importante, los balnearios se significan por la permanencia del cliente en las instalaciones del complejo. Esta es una característica diferencial importante. En sus entrevistas a los propietarios/gerentes (mejor en femenino) de establecimientos rurales, SPARRER (2007) destacaba 
cómo estas responsables preferían en general que sus clientes parasen poco tiempo en la propiedad debido a la necesidad de los dueños de complementar la atención de los usuarios con la realización de otras tareas, como las propias del hogar u otro trabajo remunerado. Frente a esta situación que se detallaba hace unos años, la oferta termal es mucho más cerrada de tal forma que los huéspedes pasan la mayor parte del tiempo dentro del establecimiento, generando poca dinámica social y económica en el entorno.

En un pequeño trabajo presentado a un congreso internacional de turismo termal que tuvo lugar en Ourense en marzo de 2011, FERNÁNDEZ VILA (2011) expuso los resultados de una investigación sobre el recuperado balneario de Guitiriz, localizado en una zona rural de la provincia de Lugo. Entre las conclusiones de la autora del estudio estaba que aproximadamente el 95\% del gasto efectuado por los clientes quedaba dentro del establecimiento; igualmente estimó que las empresas del entorno solo se benefician de un $10 \%$ del montante total del gasto efectuado por el balneario para servicios y abastecimiento. Obviamente sí hay elementos positivos como la creación de empleo, sobre todo joven y femenino, si bien falta por determinar la calidad del mismo.

Por otra parte, el año santo de 2010 abre un paréntesis hasta 2021 sin un nuevo Xacobeo. Todavía no se sabe muy bien qué consecuencias tendrá sobre las políticas públicas y, en definitiva, sobre su mantenimiento como elemento central de la oferta en Galicia. De momento los resultados provisionales de 2011 muestran la persistencia de un elevado número de peregrinos, no tantos como en 2010, aunque similares en volumen a los del año santo de 2004. Desconocemos si este número se corresponde a caminantes que retrasaron su viaje en 2010, o si realmente responde a la consolidación del producto. Las acciones promocionales descendieron notablemente por causa de los recortes presupuestarios así como por no ser año santo. Habrá que esperar para comprobar cómo evoluciona el interés por los caminos de Santiago cuyo impacto sobre el mundo rural gallego, aun siendo menor del esperado, es puntualmente significativo.

Por último, merece una reflexión final la situación de la organización territorial en relación a las consecuencias sobre el desarrollo de los turismos de interior. La fuerte centralización actual empieza a ser un lastre para la vocación turística de muchas comarcas. Hasta el momento los criterios dominantes para el impulso del turismo desde la administración autonómica se centran fundamentalmente en tipologías de la oferta. Con excepción de los programas en destino no hubo un interés particular por dar un mayor protagonismo a las comarcas. Incluso existió una significativa confusión en cuanto a la pertenencia de los municipios a unas u otras entidades. Esta evidencia se 
advierte también en espacios más o menos definidos y que tienen una interesante trayectoria en lo relativo al turismo, como son los casos de Costa da Morte y Ribeira Sacra.

La nueva Ley de Turismo publicada en el Diario Oficial de Galicia a finales de 2011, lejos de poner remedio a esta situación introduce, a nuestro juicio, mayor confusión. Esta afirmación se sustenta en los artículos 23, 24 y 27 de la citada ley. En el primero de ellos se habla de los geodestinos, viejo concepto ahora recuperado y que hace alusión a áreas homogéneas con una identidad turística singular; su protagonismo estará en el ámbito de la promoción. El artículo 24 está dedicado a los territorios de preferente actuación turística, pero que no tienen que ser coincidentes con ninguno de los citados geodestinos. Finalmente se mantiene también la figura, pensamos que inútil, de los municipios turísticos.

Es cierto que el desarrollo de la ley puede mejorar mucho su contenido inicial. Sin embargo, y en base a los antecedentes existentes, nos tememos que no se van a solucionar algunos problemas que ya resultan muy persistentes. Entre ellos la ausencia de entes gestores comarcales público-privados que tengan la capacidad, en coordinación con la administración autónomica y con el resto de las comarcas, de desarrollar sus estrategias turísticas propias. Esto resultaría fundamental para el impulso de los turismos de interior, en la medida en la que estos territorios dependerían más de sus capacidades para generar dinámicas y menos de las decisiones de otros.

Parece claro que el futuro de los turismos de interior en Galicia es bastante incierto y pasa fundamentalmente por una dinamización previa de estos territorios. Consideramos que es un error pretender que sea el turismo quien tire del desarrollo. Al contrario, deberían ser la agricultura y la ganadería los pilares para crear una sociedad emprendedora y unos espacios vivos en los que se engarcen otras actividades complementarias como el turismo. Este camino es el que falta por iniciar y sirve para entender el fracaso del denominado turismo rural. Otras fórmulas como el termalismo o el de los hoteles monumento no dejan de ser, en la mayor parte de los casos, implantaciones ajenas que utilizan estas áreas interiores como soporte y decorado de nuevas funciones que se desarrollan fundamentalmente, o bien dentro de los propios establecimientos, o bien en zonas urbanas o costeras próximas.

\section{BIBLIOGRAFIA}

ABAD, P. (2003): «El turismo rural en Galicia», Revista Galega de Economía, vol 12 (2), pp 5-28.

ALÉN, E. y FraIZ, J. (2006): «Evaluación de la relación existente entre la calidad de servicio, la satisfacción y las intenciones de comportamiento en el ámbito del tu- 
rismo termal». En Revista Europea de Dirección y Economía de la Empresa, Vol 15 (3), 171-184.

AzEvedo, M.; AzEvedo, J. (Eds.), (2002): Turismo, o desafio da sustentabilidade. São Paulo, Ed. Futura.

BARDÓN, E. (1987): «Turismo rural en España: algunas iniciativas públicas». En Estudios Turísticos, $\mathrm{n}^{\circ}$ 94, 63-76.

BesteIRo, B. (2006): «El turismo rural en Galicia. Análisis de su evolución en la última década». En Cuadernos de Turismo, n $17,25-49$.

BotE, V. (1984): «El turismo rural en España: una estrategia artesanal para una ocupación responsable del espacio». En Revista Tecno-Hotel, $\mathrm{n}^{\circ} 238$.

Bote, V. (1985): «Plan de acción sobre conservación y desarrollo de los recursos turísticos de la comarca de La Vera (Cáceres)». En Estudios Turísticos, nº 88, 51 64.

Bote, V. (1987): «Importancia de la demanda turística en espacio rural en España». En Estudios Turísticos, n $^{\circ}$ 93, 79-91.

Bote, V. (1988): Turismo en espacio rural. Rehabilitación del patrimonio sociocultural y de la economía local. Madrid, Ed. Popular.

CABAlaR, M. (2010): O uso público nos espazos naturais protexidos de Galicia. Diagnose e propostas de acción. Tesis Doctoral inédita, Departamento de Geografía, Universidad de Santiago de Compostela.

CAls, J.; CAPEllà, J.; VAQUÉ, E. (1995): El turismo en el desarrollo rural de Espa$\tilde{n} a$. Madrid, Ministerio de Agricultura, Pesca y Alimentación.

CÀnOVES, G.; Herrera, L.; VILlarino, M. (2005): «Turismo rural en España: paisajes y usuarios, nuevos usos y nuevas visiones». En Cuadernos de Turismo, ${ }^{\circ}$ $15,63-76$.

CÀnoves, G.; Villarino, M.; Herrera, L. (2006): «Políticas públicas, turismo rural y sostenibilidad: difícil equilibrio». En Boletín de la AGE, nº41, 199-217.

Crosby, A. (1993): El desarrollo turístico sostenible en el medio rural. Madrid, CEFAT.

Del Moral, J. (s.f.): «Contribución socio-económica de las aguas minerales en la Comunidad Autónoma de Galicia» En http://aguas.igme.es/igme/publica/pdfart3/ contribucion.pdf.

FERNÁDEZ VILA, F. (2011): «Evolución e Guitiriz como destino termal: unha comparativa entre dous modelos de desenvolvemento turístico». Comunicación oral presentada al Congreso Internacional de Turismo Termal, Ourense, 2011.

Flores, T.; Bote, V. (Dirs.), (1987): Turismo en el medio rural. Oviedo, Servicio de Publicaciones del Principado de Asturias.

FORNEAU, F. (1984): «Realidades y posibilidades del turismo rural en la Sierra Norte de Sevilla», Estudios Turísticos, n ${ }^{\circ}$ 2, 27-38.

Galiano, E. (1991): «El turismo rural en España». En Estudios Turísticos, n ${ }^{\circ} 10$, 39-46.

GIL, C.; Fraiz, J.; GuEIMONDE, A. (2010): «Turismo creativo a través del patrimonio cultural inmaterial, una propuesta para el turismo rural en Galicia». En Retos para el turismo español. Cambio de paradigma. Actas del XIV Congreso AECIT, Gijón, 353-373. 
GRANDE, J. (2010): «Diálogo turismo rural-turismo urbano. Apuntes para una reflexión». En Retos para el turismo español. Cambio de paradigma. Actas del XIV Congreso AECIT, Gijón, 633-643.

INFORMES de los caminos de Santiago. Varios (2007-2008) Santiago, Centro de Estudos Turísticos de la USC-Xunta de Galicia. No publicados.

LOIS, R.; LAGE, M.; ÁlVAREZ, S. (coord.), (2002): Turismo rural e desenvolvemento territorial. Ed. Ayuntamiento de Arzúa.

LOIS, R.; PIÑEIRA, M.; SANTOMIL, D. (2009): «Imaxe e oferta de aloxamento no medio rural de Galicia». En Revista Galega de Economía, vol. 18 (2), 71-90.

LOIS, R.; SANTOS, X. (2004): «Planificación y espontaneidad en el desarrollo rural». En Rodríguez, R. y PÉREZ, E. (Coords.): Espacios y desarrollos rurales. Gijón, Ediciones Trea, 131-156.

MARTíneZ Moure, O. (2008): «El Programa de Termalismo Social del IMSERSO: la promoción del turismo activo en la tercera edad a través del agua. El caso de Galicia». En Medicina Naturista vol. 2 (2), 143-147.

Martínez Roget, F. (2004): Turismo rural en Galicia. Contribuciones al desarrollo sostenible. Santiago, Xunta de Galicia.

PAÜL, V. (2009): «El turismo en el parque natural do Invernadeiro. Situación heredada y perspectivas de cambio». En Revista Cuadernos de Turismo, n 24, 135 167.

PÉREZ FrA, M.; LÓPEZ IGLESIAS, E. (2005): «La contribución del turismo a la diversificación de actividades en un espacio rural periférico. Análisis del impacto de la iniciativa Leader en Galicia». En Estudios Agrosociales y Pesqueros, n 206, 111-135.

REYNA, S. (Dir.), (1992): El turismo rural en el desarrollo local. Madrid, Ministerio de Agricultura, Pesca y Alimentación.

RoDRÍGUEZ, R.; PÉREZ, E. (Coords.): Espacios y desarrollos rurales. Gijón, Ediciones Trea.

SPARRER, M. (2007): Turismo no espazo rural e desenvolvemento. Santiago, Xunta de Galicia.

VAlENZUEla, M. (Coord.), (1997): Los turismos de interior. El retorno a la tradición viajera. Madrid, Ediciones de la Universidad Autónoma de Madrid.

VerA, F. (Coord.), (2011): Análisis territorial del turismo y planificación de destinos turísitcos. Valencia, Tirant Lo Blanch.

www.ietgalicia.com

www.ine.es

www.termatalia.com

www.turgalicia.es 